\title{
First-stage clear-cell endometrial carcinoma with paraaortic lymph node recurrence: a case report
}

\author{
Strahil Asenov Strashilov ${ }^{1}$, Stanislav Slavchev ${ }^{2}$, Stoyan Kostov ${ }^{2}$, Angel Yordanov ${ }^{1}$ \\ ${ }^{1}$ Medical University of Pleven, Bulgaria \\ ${ }^{2}$ Medical University Varna, Bulgaria
}

\begin{abstract}
Introduction: Endometrial cancer (EC) is the sixth most common malignancy in women worldwide; in developed countries this oncological entity climbs to fourth place. In 2012 worldwide 319600 new cases were registered and about 1/3 of those were diagnosed in Europe, i.e. more than 100000 cases. Statistics in Bulgaria show an increase in the incidence; the National Cancer Registry reported an incidence rate of $8.6 \%$ for 2013 , with 1293 new cases; about 300 women die because of this diagnosis every year. Clear-cell endometrial carcinoma (EC) is relatively rare, in 1-6\% of cases, with significantly shorter survival rates even for early stages (I and II), compared to the other histological subtypes.

Case report: A 62-year-old patient was subjected to surgery for clear-cell endometrial carcinoma, stage pT1NOMO, FIGO stage IA. Despite radical surgical treatment as per Bulgarian oncological standards - total hysterectomy with bilateral adnexectomy and pelvic lymph node dissection with postoperative radiotherapy there was a recurrence in a the paraaortic lymph node 15 months later. A second surgical intervention with radical resection of the parailiac metastatic lymph nodes on the left was done with subsequent pseudo-adjuvant platinum-based chemotherapy, and no further relapse has occurred 36 months later.

Conclusions: Clear-cell EC is an aggressive disease with worse prognosis than the endometroid subtype. Therefore, comprehensive surgery and surgical staging should be performed, including pelvic and paraaortic lymph node dissection, regardless of stage.
\end{abstract}

Key words: clear-cell endometrial carcinoma, adjuvant treatment, surgical management.

\section{Introduction}

Endometrial cancer (EC) is the sixth most common malignancy in women worldwide; in developed countries this oncological entity climbs to fourth place. In 2012 worldwide 319600 new cases were registered, and about $1 / 3$ of those were diagnosed in Europe, i.e. more than 100000 cases [1]. Statistics in Bulgaria show an increase in the incidence: The National Cancer Registry reported an incidence rate of $8.6 \%$ for 2013 , with 1293 new cases; about 300 women die because of this diagnosis every year. The most frequent histological subtype is endometroid EC - reported in about 75-80\% of cases, followed by serous papillary in 15-20\%, whereas the clear-cell histology was reported in only 1-6\% [2]. Overall survival differs significantly depending on histology subtype even in the early (I and II) stages and is significantly better for the endometroid compared to the clear-cell EC [3].

\section{Case report}

A 62-year-old woman in natural menopause for 12 years presented to the Department of Gynaecological Oncology of the University Hospital "Dr. Georgi Stranski", Pleven, Bulgaria with complaints of vaginal bleeding. She reported having diabetes mellitus type II with obesity first degree (BMI - 32.65), arterial hypertension, and glaucoma; no previous surgeries. She reported having 2 normal deliveries and 1 spontaneous abortion. A standard work-up was initiated with clinical and gynaecological examination. The gynaecological examination established enlarged uterus as in 2-month pregnancy with no pathology findings in vagina, adnexa, or parameter ligaments. Gynaecological ultrasound measured a 10-mm endometrial mucosa. She was subjected to dilatation and curettage and a diagnosis of clear-cell EC was histologically confirmed. Staging of the disease with chest radiograph, abdominal and pel- 


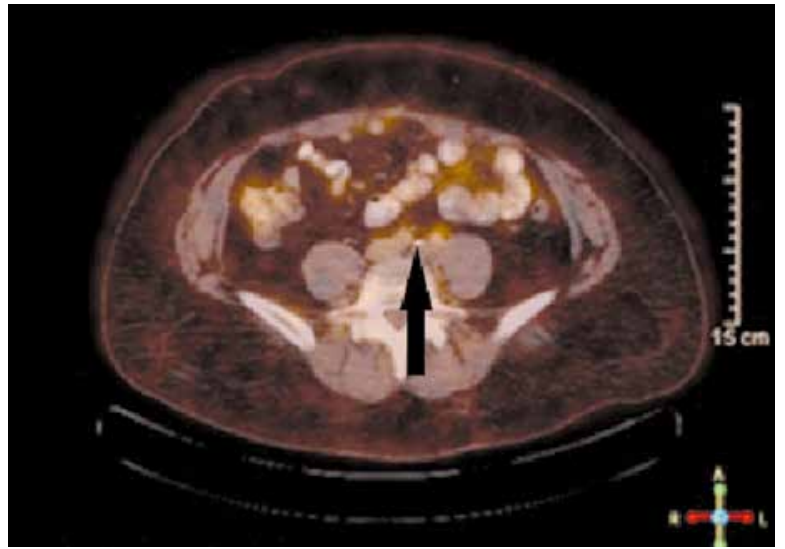

Fig. 1. PET/CT image of the metastatic lymph node in the common iliac lymph node chain

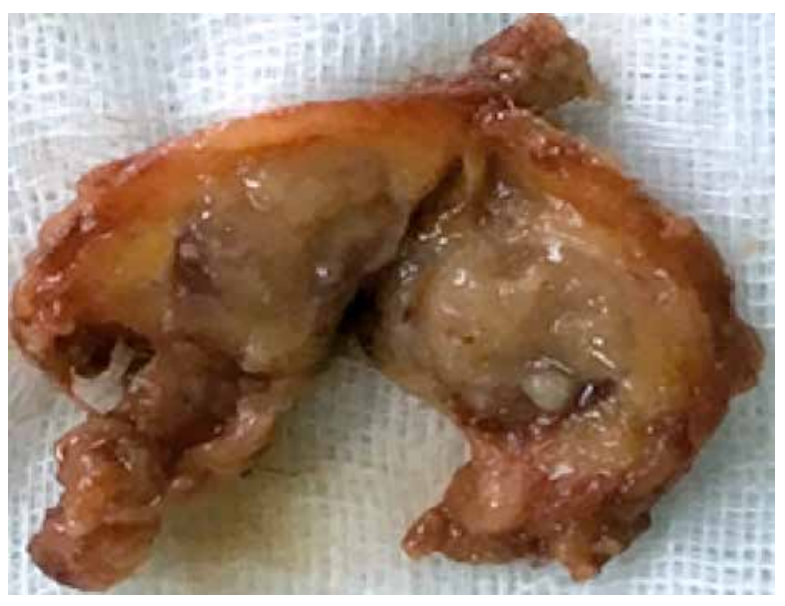

Fig. 2. Surgically resected conglomerate of iliac lymph nodes

vic ultrasound, followed by computed tomography (CT) showed no abnormal findings or enlarged lymph nodes (LNs). Thus, the disease was classified as CT1NOMO. The oncological committee referred the patient to surgery. A total hysterectomy with bilateral adnexectomy, pelvic LN dissection, and infracolic omentectomy with peritoneal lavage was done as a standard surgical approach. The final histology described low-proliferative endometrium with intramucosal and polypoid clear-cell EC without infiltration in the myometrium, the cervical channel or the isthmus; there was no lymphovascular invasion; 14 dissected LNs as well as the annexes and the omentum were free of metastases. FIGO staging was thus confirmed as $1 \mathrm{~A}$ and surgical staging was pT1A pNO MO. The oncological committee referred the patient to intravaginal brachytherapy postoperatively, and a total dose of 30 Gy in 6 weeks with daily dose of 5 Gy was delivered using iridium 192. The patient was thereafter referred to follow-up (FU) in the outpatient setting. FU visits were organized initially every 3 months and consisted of clinical examination, laboratory tests, and abdominal and pelvis ultrasound. A chest radiograph was done annually.
During the first 12 months there were no signs of disease recurrence. Because the patient reported having a ventral hernia at month 15 after surgery, a surgical correction was planned after check-up. A CT of abdomen and pelvis was done with a solitary enlarged $\mathrm{LN}$ in the region of the left common iliac artery, measuring 19/13 mm. A confirmatory PET/CT showed no other secondary sites (Fig. 1). The metastatic burden was considered as low and radically resectable. As an oligo-metastatic first progression with only one LN involved, a surgical approach was considered: elective dissection in the region of the common iliac artery to the left followed by correction of the hernia via hernioplasty with a mesh. A conglomerate of 6 common iliac LNs was dissected (Fig. 2), and the final histology report confirmed a metastasis of clear-cell EC in only one of them. Six cycles of pseudo-adjuvant chemotherapy with carboplatin and paclitaxel were done. No serious adverse events were reported, and the patient was again referred to FU. Regular CT of abdomen and pelvis with i.v. contrast enhancement was done every 3 months. Currently, at the end of February 2020 - 36 months after first disease progression - the patient is free of disease with ongoing clinical and imaging complete remission.

\section{Discussion}

The aetiology of the clear-cell endometrial carcinoma $(E C)$ is unclear, but until present it is considered to be different from endometroid subtype. A specific histological putative precursor lesion (atypical glandular lesions and specific IHC constellation) in the mucosa in about $90 \%$ of patients with clear-cell cancer is described, and it was absent in benign conditions and in the endometroid EC subtype [4]. A relatively high incidence of clear-cell EC is registered in older patients, women treated with tamoxifen for breast cancer, and in cases with pelvic radiotherapy (most typically for other malignancies) $[5,6]$.

Symptoms, as in all EC, most frequently consist of vaginal bleeding despite menopause; changes in the cytological smear may also be an initial alert in asymptomatic patients, but the diagnosis requires histological confirmation [7]. Ultrasound may measure the endometrium below $5 \mathrm{~mm}$ in about $35 \%$ of the cases [8], which is a limitation of the ultrasound screening in menopausal women [9]. In our case, the ultrasound measured $10 \mathrm{~mm}$ of endometrium mucosa, but the cytological smear showed no atypical cells. As largely described the literature, the co-morbidities of the patient (diabetes mellitus type II and obesity) are established as risk factors for EC.

Because clear cell EC is a rare condition, the postoperative management may be subjected to discussion [10]. As an initial and potentially radical management, surgery is crucial [11]. Even if there is no invasion in the 
myometrium, this cancer subtype is more aggressive, with high metastatic potential and significant risk of extrauterine spread. Preoperative clinical comprehensive staging of regional $L N$ and systemic spread is also essential. Many systems, using different factors, try to predict preoperatively the potential presence or absence of $L N$ involvement $[12,13]$ or the risk of recurrence [14], but none is definitive. The sentinel LN approach in EC is also studied [15], but there are many different methods described and this procedure remains controversial for EC in general. This is the reason why surgery consists of total hysterectomy with both annexes, pelvic and paraaortic $L N$ dissection, and resection of all potentially suspicious lesions bilaterally. Peritoneal cytology is recommended to exclude microscopic spread, invisible to the eye. It is important to mention that in about $52 \%$ of cases, the disease preoperatively is considered as only limited to the uterus but intraoperatively an extrauterine spread is diagnosed [16]. The role of the omentectomy is debatable and different professional societies disagree - the ESGO does not consider it as routine mandatory procedure [17] whereas the NCCN does [18]. In our institution, in clear-cell cancer we consider omentectomy as a staging surgical procedure and we recommend it up to the level of the transverse colon.

The postoperative management is also subject to discussions. Postoperative radiotherapy may be indicated $[19,20]$, but more recent research does not register improvement in relapse-free or overall survival of patients with clear-cell EC [10]. Additionally, due to its aggressive behaviour and the tendency for early recurrences, most specialists discuss adjuvant platinum-based chemotherapy in the postoperative management of all cases of clear-cell EC [10].

In our case, adjuvant chemotherapy was not initially undertaken despite the aggressive tumour biology and the large tumour board discussions. The disease was regarded as early stage, and only postoperative brachytherapy was considered. Pseudo-adjuvant chemotherapy was done only after radical resection of the small tumour burden relapse, confirming the aggressive tumour biology. In contrast to our experience, the national guidelines in Bulgaria do not recommend adjuvant chemotherapy in early stages, regardless of histology. This case also exemplifies the role of adjuvant chemotherapy in clear-cell EC, but currently in Bulgaria it may be considered only after case-by-case MDT discussion.

Staging of regional LNs is definite after surgery and pathological examination of dissected LNs. In clear-cell $E C$, dissection of the paraaortic and paracaval LNs is mandatory because they are considered regional. In our case, the preoperatively negative CT led to the omission of paraaortic and paracaval LN dissection. Despite the pelvic LNs being histologically free from metastasis, we consider that LN dissection in paraaortic and paracaval regions is mandatory. Because there are limitations in the preoperative clinical staging via $\mathrm{CT}$, the assessment of regional LNs rely on size and might miss or underestimate (micro)metastases in LNs with normal size. Additionally, the absence of metastases in pelvic LNs does not exclude further dissemination in other regional $L N$ groups - involvement of paraaortic LNs only (without pelvic LNs) is reported in $16 \%$ of cases [21].

\section{Conclusions}

Clear-cell EC has aggressive clinical behaviour, and the prognosis is relatively poor in comparison to endometrioid EC subtype. Clear-cell EC has significant metastatic potential even in the early stages when the disease is only in the endometrium. Comprehensive surgery with dissection of all groups of regional lymph nodes (LNs), including paraaortic LN dissection, should always be done with subsequent discussion of adjuvant modalities such as chemo- and radiotherapy. Detection of early and localized asymptomatic relapses may remain subject to local FU protocols, but because it may lead to management (e.g. surgery or radiosurgery) with curative intent despite the aggressive tumour biology, all women with clear-cell EC should be subjected to close and sophisticated clinical and imaging FU.

\section{Disclosure}

The authors report no conflict of interest.

\section{References}

1. Torre LA, Bray F, Siegel R, et al. CA Cancer J Clin 2015; 65: 87-108.

2. Abeler V, Kjørstad K. Clear cell carcinoma of the endometrium: a histopathological and clinical study of 97 cases. Gynecol Oncol 1991; 40: 207-217.

3. Malpica A, Tornos C, Burke T, et al. Low-stage clear-cell carcinoma of the endometrium. Am J Surg Pathol 1995; 19: 769-774.

4. Fadare O, Liang S, Ulukus E, et al. Precursors of endometrial clear cell carcinoma. Am J Surg Pathol 2006; 30: 1519-1530.

5. Magriples U, Naftolin F, Schwartz P, et al. High-grade endometrial carcinoma in tamoxifen-treated breast cancer patients. J Clin Oncol 1993;11: 485-490.

6. Hoffman K, Nekhlyudov L, Deligdisch L. Endometrial carcinoma in elderly women. Gynecol Oncol 1995; 58: 198-201.

7. Gu M, Shi W, Barakat R, et al. Pap smears in women with endometrial carcinoma. Acta Cytol 2001;45: 555-560.

8. Wang J, Wieslander C, Hansen G, et al. Thin endometrial echo complex on ultrasound does not reliably exclude type 2 endometrial cancers. Gynecol Oncol 2006; 101: 120-125.

9. Scioscia M, Noventa M, Laganà AS. Abnormal uterine bleeding and the risk of endometrial cancer: can subendometrial vascular ultrasound be of help to discriminate cancer from adenomyosis? Am J Obstet Gynecol 2020; S0002-9378(20)30605-0.

10. Olawaiye A, Boruta D. Management of women with clear cell endometrial cancer. Gynecol Oncol 2009; 113: 277-283.

11. Creasman W, Kohler M, Odicino F, et al. Prognosis of papillary serous, clear cell, and grade 3 stage i carcinoma of the endometrium. Gynecol Oncol 2004; 95: 593-596.

12. Cignini P, Vitale SG, Laganà AS, et al. Preoperative work-up for definition of lymph node risk involvement in early stage endometrial cancer: 5-year follow-up. Updates Surg 2017; 69: 75-82. 
13. Benati $M$, Montagnana $M$, Danese $E$, et al. Aberrant telomere length in circulating cell-free DNA as possible blood biomarker with high diagnostic performance in endometrial cancer. Pathol Oncol Res 2020; 10.1007/ s12253-020-00819-x.

14. Casarin J, Bogani G, Serati M, et al. Presence of glandular cells at the preoperative cervical cytology and local recurrence in endometrial cancer. IntJ Gynecol Pathol 2019; 10.1097/PGP.0000000000000642.

15. Rossetti D, Vitale SG, Tropea A, et al. New procedures for the identification of sentinel lymph node: shaping the horizon of future management in early stage uterine cervical cancer. Updates Surg 2017; 69: 383-388.

16. Thomas M, Mariani A, Wright J, et al. Surgical management and ad juvant therapy for patients with uterine clear cell carcinoma: a multiinstitutional review. Gynecol Oncol 2008; 108: 293-297.

17. https://www.esgo.org/explore/guidelines/

18. https://www.nccn.org/professionals/physician_gls/default.aspx.

19. Stewart K, Martinez A, Weiner S, et al. Tenyear outcome including patterns of failure and toxicity for adjuvant whole abdominopelvic irradiation in high-risk and poor histologic feature patients with endometrial carcinoma. Int J Radiat Oncol Biol Phys 2002; 54: 527-535.

20. Smith R, Kapp D, Chen Q, et al. Treatment of high-risk uterine cancer with whole abdominopelvic radiation therapy. Int J Radiat Oncol Biol Phys 2000; 48: 767-778

21. Li B, Li X, Wu L, et al. A pilot study of sentinel lymph node identification in patients with endometrial cancer. Bull Cancer 2007; 94: E1-4. 